\title{
CONVERGENCIA DE NORMAS INTERNACIONALES DE AUDITORÍA Y ASEGURAMIENTO EN LAS PYMES COLOMBIANAS Y SU RELACIÓN CON LOS RIESGOS ASUMIDOS POR LOS AUDITORES
}

\section{CONVERGENCE OF INTERNATIONAL STANDARDS ON AUDITING AND ASSURANCE IN COLOMBIAN SMES AND THEIR RELATION TO THE RISKS ASSUMED BY THE AUDITORS}

\author{
CONVERGÊNCIA DAS NORMAS INTERNACIONAIS DE \\ AUDITORIA E ASSURANCE NAS PME COLOMBIANAS E SUA \\ RELAÇÃO COM OS RISCOS ASSUMIDOS PELOS AUDITORES
}

Por: Adriana Patricia Leuro Carvajal

Doctoranda en Gestión, Universidad Escuela de Administración de Negocios, EAN, Colombia. Docente Departamento de Contaduría, Universidad Central. Email: aleuroca1117@ean.edu.co, Colombia.

\section{RESUMEN}

El artículo reflexiona sobre el proceso de convergencia de las normas internacionales de auditoría y de aseguramiento, enfatizando en las Pequeñas y medianas empresas de Colombia, en adelante Pymes, y su relación con los riesgos asumidos por los auditores internos y externos. Revisa los roles legítimos establecidos por la Administración de Riesgos Empresariales - ERM, como son la evaluación de información y la consultoría de empresas. Profundiza en la observancia del código de ética de IFAC y sus principios fundamentales, relacionando la idoneidad del auditor como empresario y el impacto que el concepto pudiera tener en las organizaciones empresariales. Para este fin, fueron revisados textos de IFAC, IASB, COSO e INTOSAI. Enseguida, se proponen conclusiones con base en el análisis de la información observada, mencionando asuntos que las Pymes pudieran tener en cuenta al asumir un proceso de convergencia, igual se aportan ideas para robustecer la implementación de las diferentes etapas del proceso en las compañías que lo quieren aplicar y plantea tareas que deberían realizar los auditores. Esto a fin de disminuir la asunción de riesgos a los que pudieran estar expuestas las empresas de auditoría en sus roles de evaluación y consultoría, y de las empresas que las contratan.

Palabras claves: Pymes, Convergencia y aseguramiento, Riesgo, Auditor.

JEL: M4 


\begin{abstract}
The article focus on the convergence of international standards of auditing and assurance, emphasizing small and medium-sized Colombia, hereinafter SMEs, and its relation to the risks assumed by internal and external auditors. Check legitimate roles established by the Enterprise Risk Management - ERM, such as evaluation of information and consultancy firms. Delves into compliance with IFAC Code of Ethics and its fundamental principles relating the suitability of the auditor as an entrepreneur and the impact it could have on the concept of business organizations. To this end, they were revised texts of IFAC, IASB, COSO and INTOSAl. Then, conclusions are proposed based on the analysis of the observed data, mentioning issues that SMEs might consider assuming a convergence process, just ideas to strengthen the implementation of the various stages in the companies that provide They want to apply and poses tasks to be performed by the auditors. This in order to reduce the risk taking that may be exposed audit firms in their roles as evaluation and consulting, and companies that hire.
\end{abstract}

Keywords: SMEs, Convergence and assurance, Risk, Auditor.

JEL: M4

\title{
RESUMO
}

O artigo enfoca a convergência das normas internacionais de auditoria e garantia, enfatizando pequenas e médias Colômbia, a seguir as PME, e sua relação com os riscos assumidos pelos auditores internos e externos. Verifique papéis legítimos estabelecidos pela Enterprise Risk Management - ERM, como a avaliação de informação e consultoria em empresas. Investiga o cumprimento IFAC Código de Ética e seus princípios fundamentais sobre a adequação do auditor como um empreendedor e impacto que isso poderia ter sobre o conceito de organizações empresariais. Para este fim, eles foram revisados textos da IFAC, o IASB, COSO e INTOSAI. Em seguida, as conclusões são propostas com base na análise dos dados observados, mencionando problemas que as PME podem considerar assumindo um processo de convergência, apenas idéias para fortalecer a implementação dos vários estágios nas empresas que fornecem Eles querem aplicar e coloca tarefas a serem desempenhadas pelos auditores. Esta, a fim de reduzir a assunção de riscos que podem ser expostas as empresas de auditoria em seus papéis como a avaliação e consultoria, e as empresas que os contratam.

Palavras-chave: PME, Convergência e garantia, Risco, Auditor.

JEL: M4 
Adriana Patricia Leuro Carvajal

Convergencia de normas internacionales de auditoría y aseguramiento en las pymes colombianas y su relación con los riesgos asumidos por los auditores

\section{INTRODUCCIÓN}

Con base en la información consultada en la página web de IASB, varios países de América Latina, entre ellos Colombia, han decidido aceptar la propuesta de convergencia internacional de normas en temas contables, de información financiera, de aseguramiento de información y XBRL y otras normas, hecha por las entidades supranacionales. Para tal fin, los países están abordando normativa de la Federación Internacional de Contadores (International Federation of Accountants - IFAC), y la Junta de Normas Internacionales de Contabilidad (International Accounting Standards Board - IASB).

En tal sentido, las naciones, los empresarios y los auditores se han interesado en conocer y profundizar en los objetivos promovidos por dichas entidades. Ellas buscan asesorar a los contadores profesionales, proteger a los inversionistas y servir al interés público.

Para servir al interés público, la Junta de estándares Internacionales de Auditoría y Aseguramiento (IAASB), emite las normas internacionales de auditoría y aseguramiento de alta calidad, reconoce que las normas deben ser comprensibles, claras y susceptibles de aplicación coherente. Estos aspectos de la claridad sirven para mejorar la calidad y la uniformidad de la práctica en todo el mundo. (IFAC, 2015).

Por tal razón, en el año 2009, IFAC a través de la Junta de estándares de auditoría y aseguramiento (IAASB, por sus siglas en inglés) crea un Proyecto denominado "Claridad" el cual tuvo por objeto revisar, modificar y reformular las NIAS y la International Standard on Quality Control1 (En adelante ISQC1), a fin de crear mensajes homogéneos para los usuarios de las mismas, aunque su trabajo ya terminó, IAASB ha seguido liderando la actualización de normas de manera permanente, ejemplo de ello es la "Guía de Control de Calidad para Pequeñas y Medianas Firmas de Auditoría”, un texto en inglés, en su tercera edición, publicada en 2011 por IFAC. El texto de la guía en español, va en la tercera edición y se publicó en 2012; su título original fue: Guide to Quality Control for Small and Medium-SizedPractices. (Bernal, 2010).

En concordancia y para cubrir las exigencias de estas entidades supranacionales, Colombia ha emitido normas como las leyes 1474 de 2011 y 1314 de 2009 y los decretos reglamentarios 2706, 2784 de 2012, 1851, 3019, 3022,3023 y 3024 de 2013, 2548 de 2014 y 0302 de 2015, con el objeto de normalizar el plan de convergencia y asirse a los nuevos lineamientos. Lo anterior, en coherencia con las Normas internacionales de Auditoría, especialmente la NIA 240, la cual establece que la responsabilidad del auditor es considerar el fraude y el error en una auditoría de estados financieros, lo cual hace parte de la estructura administrativa y del control del Estado; la NIA 200 y la Norma Internacional de Control de Calidad - NCC1 (ISQC1, por sus siglas en inglés) que establecen los requerimientos mínimos para realizar una auditoría con calidad.

Por ende, el alcance de las normas que regulan la convergencia incluye las Pymes, lo que exige por tanto, asignar y presupuestar recursos para llevar a cabo 
dicho proceso. También propone revisar entidades que pudieran generar ideas similares en temas equivalentes como la Organización Internacional de Entidades Fiscalizadoras Superiores - INTOSAI.

En consecuencia, a continuación se presenta un análisis y evaluación de la convergencia de Normas Internacionales de Auditoria y Aseguramiento en las Pymes, luego se revisa la relación que existe entre dichos conceptos, a continuación se analizan asuntos de las Pymes que tienen relación con los riesgos asumidos por los Auditores y por último se plantean conclusiones que podrían aportar ideas para asumir el proceso.

\section{CONVERGENCIA DE NORMAS INTERNACIONALES DE AUDITORÍA Y ASEGURAMIENTO EN LAS PYMES}

En Colombia, las Pymes están dentro del alcance de la Ley 1314 de 2009, en tal sentido, requieren acoger el proceso de convergencia de normas internacionales de información financiera y de auditoría y aseguramiento. En concordancia con la revisión de normas internacionales que incluyen los estándares de control de calidad para firmas de auditoría - NCC1 (ISQC1 por sus siglas en inglés),convergencia internacional de normas en temas contables - NIC, de información financiera NIIF, de aseguramiento de información - NIAS y Otras Normas Internacionales -ONI, de las cuales hace parte el Extensible Business Reporting Language - XBRL, definido por IFRS (2015) como "un protocolo tecnológico de información financiera", a fin de emitir reportes de información en formato homogéneo.

\subsection{Normas Internacionales de Auditoria y Aseguramiento}

En referencia, IFAC a través de la Junta de estándares de auditoría y aseguramiento (IAASB, por sus siglas en inglés) en el año 2009 creó un Proyecto denominado "Claridad" el cual tuvo por objeto revisar, modificar y reformular las normas internacionales de auditoría y aseguramiento- NIAS y la ISQC1, a fin de crear mensajes homogéneos para los usuarios de las mismas. Aunque el trabajo de este proyecto ya terminó, IAASB ha seguido liderando la actualización de normas de manera permanente, ejemplo de ello es la "Guía de Control de Calidad para Pequeñas y Medianas Firmas de Auditoría”, este es un texto en inglés, en su tercera edición, publicada en 2011 por IFAC. El texto de la guía en español, va en la tercera edición y se publicó en 2012; su título original fue: Guide to Quality Control for Small and Medium-Sized Practices.

\subsection{Convergencia en las Pymes}

Por lo anterior, "La encuesta global de 2014 IFAC le preguntó a las Pymes sobre los desafíos que enfrentan, los factores de mercado y tecnologías, que podrían afectar su futuro" (IFAC, 2015). En concordancia, Colombia emitió normas como las leyes 1474 de 2011 y 1314 de 2009 y los decretos reglamentarios 2706, 2784 de 2012, 1851, 3019, 3022,3023 y 3024 de 2013, 2548 de 2014 y 0302 de 2015, con el objeto de normalizar el plan de convergencia y asirse a los nuevos lineamientos. 
En tal contexto, fue necesario conocer el tipo de empresas que tiene Colombia, para determinar las características de las organizaciones y establecer quién y cómo requiere aplicar el proceso de convergencia; para tal fin ACOPI, tiene los siguientes datos de micro, pequeñas y medianas empresas:

La Encuesta Anual Manufacturera nos permite valorar la incidencia de la MIPYME en el panorama empresarial colombiano. Representan el 96.4\% de los establecimientos, aproximadamente el 63\% del empleo; el 45\% de la producción manufacturera, el 40\% de los salarios y el 37\% del valor agregado. Son más de 650.000 empresarios cotizando en el sistema de seguridad social (Sitio web: businesscol.com, 2015).

Por lo tanto, se hace necesario revisar el interés que pudieran tener las Pymes para aplicar el proceso de convergencia de normas internacionales y su motivación para recibir asesoría y evaluación de los auditores y consultores, ya que, de ello dependerá el éxito y la eficiencia de los resultados que se pudieran obtener. Estudios de Universia Business Review realizados en 2013 por Montoya-del-Corte, Fernández-Laviada y Martínez-García mencionan (citando a Clatworthy y Peel, 2013) que "Como puede observarse, las Pymes opinan que la auditoría, principalmente, aumenta la fiabilidad y credibilidad de la información financiera...”.

Sin embargo, no hay seguridad sobre si en Colombia las Pymes "han realizado la tarea y están realmente preparadas para actuar bajo las nuevas reglas de juego financiero y contable” (Janica, 2015). Para atender este asunto, las Cámaras de comercio y el Consejo Técnico de la Contaduría Pública (En adelante CTCP) ha realizado alianzas con universidades e instituciones educativas, aplicando campañas de capacitación que permitan a empresas y profesionales actualizarse sobre los temas necesarios para atender, planear, ejecutar y monitorear el proceso de convergencia, igual que aplicar la adecuada interpretación de las normas internacionales emitidas por el IASB y la IFAC.

Estamos entrando en un nuevo período económico en el que el éxito de las empresas y de sus marcas no es ya un éxito financiero a corto plazo pensando solamente en el inversor y el accionista, sino un éxito reputacional a medio y largo plazo pensando en el conjunto de stakeholders ya sean empleados, proveedores, clientes, accionistas y sociedad. Es un entorno competitivo, complejo y cambiante en el que los intangibles están adquiriendo un creciente protagonismo... (Amat, 2013, p. 202).

Por lo anterior, las Pymes que acepten aplicar las normas internacionales contables y financieras requieren revisar los estándares que se ajusten a su tamaño, contexto y necesidades de capacitación, a fin de evitar costos innecesarios. Por lo que será prudente evaluar la existencia de factores como: el sector económico en el que operan, el tipo de negocio que realizan, la cantidad de empleados y las políticas que cada entidad asuma en asuntos de contratación y alianzas entre otros, para determinar cuáles serán los nuevos lineamientos a aplicar. Aún así, el proceso de auditoría que deberá realizarse en las Pymes, será similar al que se pudiera 
aplicar en cualquier otra entidad, esto con base en lo establecido en las Normas internacionales de auditoría y aseguramiento.

\section{LAS PYMES Y SU RELACIÓN CON LOS RIESGOS ASUMIDOS POR LOS AUDITORES}

Por lo anterior, la NIA 240 determina que la responsabilidad del auditor es considerar el fraude y el error en una auditoría de estados financieros, lo cual hace parte de la estructura administrativa y del control del Estado Colombiano; igual que la NCC1 o ISQC1, la NIA 200, la ISSAI 1200 e ISSAI 1220 que determinan los requerimientos mínimos para realizar una auditoría con calidad y se fortalece con la Ley 43, capítulo 4 que incluye asuntos como la conciencia moral, la aptitud profesional y la independencia mental que deben asumir los contadores a la hora de realizar su trabajo.

\subsection{Las Pymes y la Ética}

En complemento, IFAC cuenta con el código de ética profesional para profesionales de la contaduría, el cual debe ser asumido de manera imperativa por todos los países y profesionales que se acojan al proceso de convergencia. Dicho código fue emitido por el consejo de normas internacionales de ética para contadores (International Ethics Standards Board of Accountants - IESBA) y promueve las buenas prácticas de ética a nivel global para los miembros de IFAC.

El código de ética de IFAC, contiene las siguientes partes:

3.1.1 Aplicación general del código: menciona y explica que los principios fundamentales de ética, son: integridad, objetividad, competencia y diligencia profesionales, confidencialidad y competencia profesional. Por lo que es deber idóneo de los contadores mantenerse actualizados; actuar sin privilegiar los intereses personales viciados; aportarle valor agregado a la profesión, a los empresarios y a la comunidad en general; generar confianza al Estado y a sus clientes, igual que guardar el debido cuidado en la práctica de su profesión.

3.1.2 Profesionales de la contabilidad en ejercicio: refiriéndose a los profesionales independientes y la forma como deben aplicar los principios fundamentales. Situación que le exige aplicar la ética y respetar su función como profesional de la contaduría.

3.1.3 Profesionales de la contabilidad en la empresa: toma en cuenta a los contadores que son contratados por las empresas para actuar en los diferentes roles organizacionales y su relación con los principios fundamentales. En tal sentido, lo hace partícipe intra-empresarial del mundo y le permite aportar ideas que sirvan al robustecimiento de las organizaciones.

\subsection{Los riesgos asumidos por los Auditores}

En concordancia, dependiendo de la naturaleza de su trabajo y la normatividad aplicada, se evidencian diferencias entre el hacer del Auditor Externo, el auditor interno y el Revisor Fiscal, ya que mientras que el primero opina sobre seguridad razonable, en Colombia el tercero opina sobre razonabilidad y continuidad del 
negocio, según preceptos del Código de Comercio, el Pronunciamiento 7 y la orientación profesional del 21 de junio de 2008, numeral 26.1 del CTCP.

Así las cosas, empiezan a generarse inquietudes respecto de cuál sería la normatividad que deberían aplicar los auditores internos que trabajan en Pymes, ya que son funcionarios que podrían ser contratados al interior de la empresa o como funcionarios independientes. En tal sentido, es necesario recordar que existe normatividad vigente como la Ley 43 de 1990, por lo tanto, si las entidades contratantes deciden aplicar normas internacionales, se interpreta que se cumplirán las normas nacionales vigentes hasta tanto sean derogadas, se adoptan las nuevas leyes y se toma la normativa internacional de manera referente.

En el caso del auditor externo, que a la luz del código de ética de IFAC es un profesional de la contabilidad en ejercicio, seguirá pautas de la NIA700 (2006), la cual establece que él opina sobre “...si ha obtenido una seguridad razonable, sobre si los estados financieros en su conjunto, se encuentran libres de desviaciones materiales, ya sea debido a fraude o error."

En referencia, Mantilla (2010) establece que seguridad razonable “...se refiere a un nivel de aseguramiento que es elevado pero no absoluto...", por lo que aunque las metodologías aplicadas por el Auditor interno, externo y el Revisor Fiscal son parecidas, cada una tiene propósitos diferentes. En tal sentido, cualquiera que sea el rol asumido por el auditor, estará obligado tanto por normas nacionales como internacionales, a mantener la independencia profesional.

Por lo anterior y teniendo en cuenta que los auditores pueden ejercer, según la metodología The Enterprise Risk Management (en adelante ERM), roles de evaluador ó consultor ante sus clientes, se deduce que los profesionales y las firmas de auditoría que la ejercen, se exponen a riesgos que pudieran tener origen en las decisiones estratégicas que asumen las organizaciones, o en eventos, amenazas surgidas en la falta de idoneidad de los empresarios, o por conflicto de intereses de los stakeholders. En tal sentido, si ocurrieran siniestros de los cuales los auditores no tuvieran conocimiento y/o que por alguna razón no fueran informados, se podrían ver impactados por el riesgo de auditoría o también denominado riesgo de detección, lo cual podría llevarlo a emitir opiniones contrarias a la realidad empresarial y por tanto a sanciones disciplinarias propias de la profesión.

En consecuencia, el auditor deberá estar atento a situaciones comunes o extraordinarias que evidencien señales de alerta, a fin de garantizar que sean reveladas en notas y/o reconocidas en estados financieros, ya que de omitirlas, podría estar compartiendo responsabilidades onerosas con la organización generadora del asunto, ya que "Los administradores y el revisor fiscal, responderán por los perjuicios que causen a la sociedad, a los socios o a terceros por la no preparación o difusión de los estados financieros..." (Ley 222 de 1995, artículo 42).

Por su parte, Mantilla (2005) menciona que las metodologías de auditoría han tenido un proceso de evolución al pasar de la revisión, a la atestación y luego al aseguramiento, y dice que “...la revisión consiste en volver a ver de nuevo; la atestación en firmar algo como cierto y el aseguramiento en privilegiar la calidad de la información por encima de todo...”. Por lo que, el auditor independiente y los 
clientes de auditoría, comparten situaciones que los pueden exponer a amenazas conjuntas. En tal sentido, ERM, respaldado por The Committee of Sponsoring Organizations of the Treadway Commission (COSO, 2014), ha emitido lineamientos sobre cómo gestionar y administrar riesgos empresariales, y además asesora al auditor sobre los roles genuinos de su labor, identificándolos como: consultores o evaluadores en las empresas a las que brinda sus servicios.

\subsection{Impacto de los riesgos en las empresas}

En atención de lo anterior, se observan escándalos empresariales como el de ENRON, la mayor empresa de energía y gas que tuvo los Estados Unidos, cuya quiebra impacto la vida de los pensionados, a los inversionistas, al entorno empresarial, a los auditores y al gobierno, entre otros, igual que promovió la necesidad de emitir la Ley Sarbanes-Oxley de 2002, a fin de robustecer la protección de los inversionistas y al interés público.

La Ley Sarbanes cambió y agregó requerimientos a las empresas, especialmente a través de las Secciones 302 y 404, creó la Junta de Supervisión sobre las Firmas Contables que dictaminan sobre los estados contables y el control interno de las compañías que cotizan públicamente. La Sección 101 de la Ley define la Junta con el nombre de Public Company Accounting Oversight Board - PCAOB, tiene la potestad entre otras atribuciones de, emitir Normativa para regular la práctica de auditoría externa frente a la Securities and Exchange Comission - SEC (Chalupowicz, 2005).

\section{Gráfico 1. Normas Internacionales de Aseguramiento que aplican en Colombia}

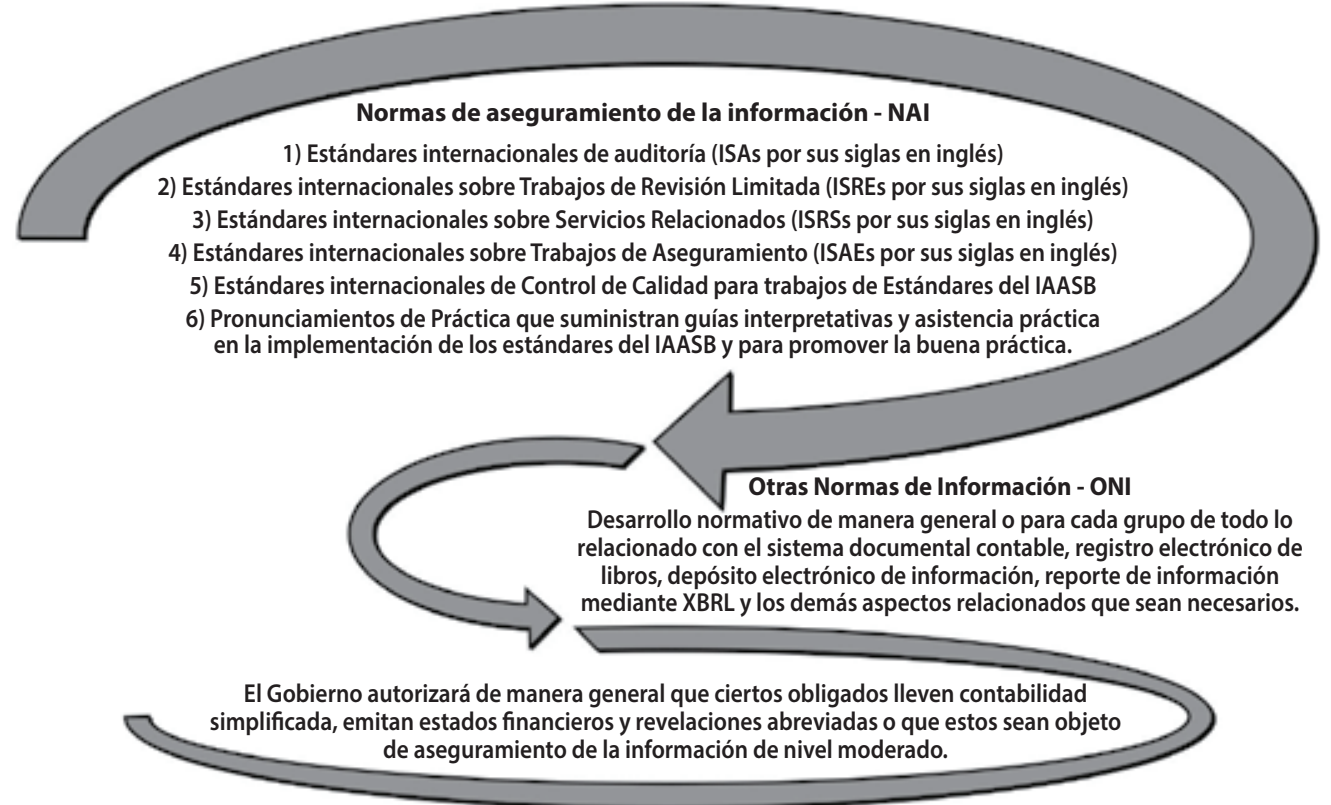

Fuente: Elaboración propia a partir del documento de direccionamiento estratégico (2012, p. 16). 
Adriana Patricia Leuro Carvajal

Convergencia de normas internacionales de auditoría y aseguramiento en las pymes colombianas y su relación con los riesgos asumidos por los auditores

Con base en lo anterior, Colombia ha creado mecanismos de convergencia que permiten adoptar, adaptar y armonizar cambios normativos internacionales incluyendo los estándares de auditoría y aseguramiento mencionados en el gráfico 1.

En este contexto:

El auditor deberá realizar un plan de convergencia desde dos aristas: una a partir de las NIC y las NIIF, en lo que tiene que ver con ser empresario, y la otra, desde las NIAS y sus normas relacionadas, en lo que corresponde a su ejercicio profesional como auditor. Esto significa, que el contador debe seguir preceptos de IASB en asuntos financieros y contables y de IAASB en temas de auditoría y aseguramiento (Leuro, 2014:95).

En consecuencia, la gerencia de las Pymes y sus auditores deberán tener claridad sobre la oportunidad en el diagnóstico, diseño, planteamiento, capacitación y ejecución de su proceso de convergencia de normas internacionales, a fin de trabajar armoniosamente e integrar grupos de interés como: los clientes de auditoría, el auditor como empresario, el contador como profesional, el Estado como guía de supervisión y control, el Revisor Fiscal como emisor de opinión, el gobierno corporativo como guía empresarial y garante de control, el comité de auditoría y los auditores como garantes de la confianza pública, con el objeto de evitar el fraude y error en la auditoría de estados financieros. Revisando claro las inhabilidades mencionadas en el artículo 48 de la Ley 43 de 1990.

En tal sentido, el Gobierno Colombiano emitió el Decreto 0302 del 20 de febrero de 2015, por el cual expidió:

El Marco Técnico normativo de las Normas de Aseguramiento de la Información (NAI), que contiene: las Normas internacionales de Auditoría (NIA), las Normas Internacionales de Control de Calidad (NICC); las Normas Internacionales de Trabajos de Revisión (NITR); las Normas Internacionales de Trabajos para Atestiguar (ISAE por sus siglas en inglés); las Normas Internacionales de Servicios Relacionados (NISR) y el Código de Ética para Profesionales de la Contaduría.

En dicho decreto se establece, que las empresas obligadas a aplicar esta norma son las empresas del “... Grupo 1, y a las entidades del Grupo 2 que tengan más de 30.000 salarios mínimos mensuales legales vigentes (smmlv) de activos o, más de 200 trabajadores...”. Lo cual incluye a las Pymes y a sus auditores.

En cumplimiento de lo anterior, la normatividad colombiana permite que las empresas que voluntariamente apliquen normas internacionales, lo podrán hacer teniendo claro que empezarían a cumplir consecuentemente con todas las exigencias vigentes en la ley. 


\section{CONCLUSIONES QUE PODRÍAN APORTAR IDEAS PARA ASUMIR EL PROCESO DE CONVERGENCIA}

Las Pymes que quieran asumir de manera impositiva o voluntaria el proceso de convergencia de normas internacionales en Colombia, deberían revisar los siguientes aspectos:

4.1 Identificar las normas nacionales exigibles en temas de contabilidad, financieros y de auditoría para la entidad interesada, con el objeto de aplicar las que sean pertinentes según el tipo de negocio y el contexto empresarial en el que se desenvuelve la empresa.

4.2 Acoger preceptos de IASB en temas contables y financieros lo cual le permitirá a las empresas y a sus profesionales, actuar de manera acorde con las exigencias legales vigentes tanto a nivel nacional como internacional.

4.3 Aplicar los estándares de IFAC en asuntos de aseguramiento, auditoría, ética profesional y otras normas internacionales, a fin de proyectar el negocio con base en lineamientos de gobierno corporativo.

4.4 Incluir en el diagnóstico y la ejecución del proceso, asuntos como:

4.4.1 Legislación vigente en el ámbito nacional e internacional.

4.4.2 Planeación de recursos financieros, humanos, tecnológicos, técnicos, de información y de financiación.

4.4.3 Gestión, medición y administración de riesgos empresariales.

4.4.4 Contratación de auditores y consultores con experiencia que guíen y asesoren el proceso de convergencia para hacer más eficientes sus procesos y resultados.

4.5 Examinar lecciones aprendidas por empresas que ya han aplicado procesos de convergencia, algunos ejemplos son: Ecopetrol y Grupo Sanitas.

4.6 Reconocer ventajas de realizar el proceso de convergencia, algunas son:

4.6.1 Unificar el lenguaje de los estados financieros en términos de exigencias nacionales e internacionales.

4.6.2 Mejorar las bases sobre las cuales se toman decisiones en las empresas.

4.6.3 Ampliar la proyección internacional de la empresa, en asuntos tales como inversión y de mercado, entre otros.

4.6.4 Robustecer la imagen contable, financiera, de aseguramiento y de control ante los stakeholders, a fin de fortalecer sus niveles de confianza y de compromiso hacia la compañía.

4.6.5 Evitar riesgos empresariales y riesgos de auditoría.

\section{LÍMITES Y FUTURAS INVESTIGACIONES}

El artículo se escribe en el contexto Colombiano, por tanto una futura investigación podría incluir otros países del ámbito Latinoamericano. 


\section{REFERENCIAS BIBLIOGRÁFICAS}

1. AMAT, J. (2013). Control 2-0. Una perspectiva del control de gestión menos financiera y más cualitativa. Profit editorial I., S.L. Barcelona España ISBN: 978-84-15505-94-5. (www.proditeditorial.com).

2. BERNAL, F. (2010). Normas Internacionales de Auditoría (NIAs) y el Proyecto Claridad. Actualidad Empresarial, № 218 - Primera Quincena de Noviembre. Recuperado de http://www.aempresarial.com/servicios/ revista/218_11.pdf

3. BUSINESSCOL (2015) Sección Pymes, Clasificación. Disponible en: http://www.businesscol.com/empresarial/Pymes/index.htm

4. CHALUPOWICZ, D. (2005). Norma de Auditoría No. 2 del PCAOB: Auditoría del control sobre la información financiera. Normaria, Boletín del Comité de Normas de Auditoría de Argentina No. 21 de 2005. Recuperado el 13 de septiembre de 2015 de https://www.iaia.org.ar/revistas/normaria/Normaria21.pdf.

5. CONSEJOTÉCNICO DE LA CONTADURÍA PÚBLICA - CTCP (2012, 5 de julio). Documento de Direccionamiento Estratégico. Disponible enwww.mincit.gov.co/descargar.php?id=66688

6. COMMITTEE OF SPONSORING ORGANIZATIONS OF THE TREADWAY COMMISSION - COSO (2014). Disponible enhttp://www.coso.org

7. FEDERACIÓN INTERNACIONAL DE CONTADORES - IFAC. (2006). NIA 700 Revisada. Recuperado de http:// www.rya.com.ve/site/attachments/article/679/31_NIA_700\%20_REVISADA\%20DICTAMEN\%20AUDITOR\%20EEFF\%20GRAL.pdf

8. FEDERACIÓN INTERNACIONAL DE CONTADORES - IFAC. (2009). ISA 240_155 Auditing international standard on auditing 240. The auditor's responsibilities relating to fraud in an audit of financial statements. Retrieved June 1, 2014 from, http://www.ifac.org/sites/default/files/downloads/a012-2010-iaasb-handbook-isa-240. pdf.

9. IFAC (2015). Proyecto claridad. Recuperado 13 de septiembre de 2015, de http://www.iaasb.org/claritycenter.

10. IFAC (2014). Encuesta Pymes 2014. Recuperado el 13 de septiembre de 2015 de www.ifac.org/publicationsresources/ifac-global-smp-survey-2014-results.

11. IFAC (2009). Código de ética para profesionales de la Contabilidad. Recuperado el 19 de septiembre de 2015 de http://www.icjce.es/adjuntos/images/pdfs/CALIDAD/codigodeeticaifaccastellanofinallogosnuevos.pdf

12. IFRS (2015). XBRL. Recuperado el 13 de septiembre de 2015, de http://www.ifrs.org/xbrl/resources/Pages/ Fundamentals.aspx

13. INTOSAI (2015). ISSAI 1200 e ISSAI 1220. Recuperado el 13 de septiembre de 2015 de: http://www.intosai. org/es/acerca-de-nosotros.html.

14. JANICA, F. (2015). Las PyME colombianas entran en la era NIIF - ¿Colombianizadas también? Recuperado el 20 de septiembre de 2015 de: http://www.comunidadcontable.com/BancoConocimiento/N/noti_20150130_ las_pyme_colombianas_entran_en_la_era_niif_colombianizadas_tambien/noti_20150130_las_pyme_colombianas_entran_en_la_era_niif_colombianizadas_tambien.asp

15. LEURO, A. (2014). El riesgo empresarial y su relación con las Normas Internacionales de Auditoría y Aseguramiento. Civilizar 9. Universidad Sergio Arboleda. Disponible en: http://revistas.usergioarboleda.edu. co/index.php/ceye/article/view/267/227 
16. MANTILLA, S. (2005). Auditoría 2005. Bogotá ECOE Ediciones. Recuperado el 11 de junio de 2014, de http:// preparatorioauditoria.wikispaces.com/file/view/De\%20la\%20Revisi\%C3\%B3n\%20al\%20Aseguramiento. pdf/102256771/De\%20la\%20Revisi\%C3\%B3n\%20al\%20Aseguramiento.pdf

17. MANTILLA, S. (2010). Aseguramiento: ¿qué es eso? Bogotá ECOE Ediciones. Recuperado el 11 de junio de 2014, de http://actualicese.com/Blogs/Expertos/SAM-aseguramiento-que-es-eso.pdf

18. MONTOYA-DEL-CORTE, J., FERNÁNDEZ-LAVIADA, A. y MARTÍNEZ-GARCÍA, F. (2014). Utilidad de la auditoría de cuentas: perspectiva de las PYMES en España. Universia Business Review. ISSN: 1698-5117., Issue 41, p. 80-97. 18p. Language: Spanish. , Recuperado el 18 de septiembre de 2015 en: Base de datos: Fuente Académica.

19. SUPERINTENDENCIA FINANCIERA (2013). Decreto 3024. Recuperado de https://www.superfinanciera. gov.co/jsp/loader.jsf?IServicio=Publicaciones\&ITipo=publicaciones\&IFuncion=loadContenidoPublicaci on\&id $=10082320$

20. SUPERINTENDENCIA FINANCIERA (2013). Decreto 3023. Recuperado de https://www.superfinanciera. gov.co/jsp/loader.jsf?IServicio=Publicaciones\&lTipo=publicaciones\&IFuncion=loadContenidoPublicaci on\&id $=10082320$

21. SUPERINTENDENCIA FINANCIERA (2013). Decreto 1851. Recuperado de https://www.superfinanciera. gov.co/jsp/loader.jsf?IServicio=Publicaciones\&ITipo=publicaciones\&IFuncion=loadContenidoPublicaci on\&id $=10082320$

22. SUPERINTENDENCIA DE SOCIEDADES (2012, 14 de marzo). Documento de Direccionamiento Estratégico. Recuperado el 1 de junio de 2014, de http://www.supersociedades.gov.co/Web/circulares\%20externas/ CIRCULAR\%20EXTERNA\%20115-0002\%20de\%202012.html.

\section{LEGISLACIÓN}

23. LEY 43 de diciembre 13 de 1990. Publicado en el Diario Oficial No. 39.602 de diciembre 13 de 1990. Disponible en: http://www.mineducacion.gov.co/1621/articles-104547_archivo_pdf

24. LEY 222 de diciembre 20 de 1995. Publicada en el Diario Oficial No. 42.156, de 20 de diciembre de 1995. Disponible en: http://www.secretariasenado.gov.co/senado/basedoc/ley_0222_1995.html

25. LEY 1314 de 2009, sobre proceso de convergencia de Normas Internacionales NIF de Auditoría y Aseguramiento. Diario Oficial No. 47.409. Disponible en: http://www.secretariasenado.gov.co/senado/basedoc/ ley/2009/ley_1314_2009.html

26. LEY 1474 de 2011. Estatuto Anticorrupción. Diario Oficial No. 48.128 de 12 de julio de 2011. Disponible en: http://www.secretariasenado.gov.co/senado/basedoc/ley_1474_2011.html

27. DECRETO 2548 de 2014. Publicado en el Diario Oficial 49.363 de diciembre 12 de 2014. Disponible en: http://www.icbf.gov.co/cargues/avance/docs/decreto_2548_2014.htm

28. DECRETO 0302 de 2015. Publicado en el Diario Oficial 49.431 de febrero 10 de 2015. Disponible en: http:// www.comunidadcontable.com/BancoMedios/Documentos\%20PDF/d-0302-15\%28mincomercio\%29.pdf. 